\title{
Severe Carpal Tunnel Syndrome Caused by Gouty Tophi Diagnosed by Dual Energy Computed Tomography: A Case Report
}

\author{
Yan GE, Fen LI, Jinwei CHEN, Jing TIAN \\ Department of Rheumatology, Second Xiangya Hospital, Changsha, China
}

\begin{abstract}
Gout is a common form of inflammatory arthritis, and the majority of gout patients experience recurrent acute attacks, joint damage, and other complications. Carpal tunnel syndrome caused by gouty tophi indicates the severity of untreated gouty tophi. In this article, we report a 54-yearold male chronic gout patient with serious carpal tunnel syndrome secondary to monosodium urate crystal deposit. The patient was admitted to our department with palmar numbness and disability for two years. He had a history of gout for 30 years but received no treatment. Multiple monosodium urate crystals were observed to be deposited in and around carpal tunnel on the three-dimensional reconstruction using dual energy computed tomography, which confirmed the diagnosis. The needle biopsy and electrophysiological test also supported the diagnosis. Our case indicates that dual energy computed tomography is a useful method to diagnose carpal tunnel syndrome associated with gout and that we should keep in mind the possibility of gouty tophi as a possible cause of carpal tunnel syndrome.

Keywords: Carpal tunnel syndrome; dual energy computed tomography; gout; monosodium urate crystal.
\end{abstract}

Gout is a common form of inflammatory arthritis and its prevalence has been increasing. ${ }^{1}$ It is believed that gout may be cured by effective urate-lowering treatment. ${ }^{2}$ However, only a minority of gout patients receive effective treatment, while the majority experience recurrent acute attacks, joint damage, and other complications around the world, particularly in developing countries.

Although not rare, carpal tunnel syndrome (CTS) caused by gouty tophi is still uncommon according to the literature, ${ }^{3}$ and its presence indicates the severity of untreated gouty tophi. ${ }^{4,5}$ In the past, it was difficult to diagnose gouty tophi induced CTS unless surgery or biopsy was performed. However, thanks to the improvements in recent years in imaging techniques such as dual energy computed tomography (DECT) and ultrasound, it is now easier to diagnose gouty tophi induced CTS. In this article, we report a chronic gout patient with serious CTS secondary to monosodium urate crystal deposit diagnosed by DECT.

\section{CASE REPORT}

A 54-year-old male patient was admitted to our department with palmar numbness and disability for two years. He had a history of gout for 30 years but received no treatment. Physical examination revealed obvious subcutaneous tophi and thenar muscle wasting. Flexion deformity of his metacarpophalangeal and interphalangeal joints is shown in Figure 1a. Positive Tinel's sign and Phalen's maneuver were present. Routine laboratory test showed high level of uric acid (608 $\mu \mathrm{mol} / \mathrm{L})$. The electrophysiological examination revealed sensory and motoring demyelinating and axon lesions in both median

Received: December 16, 2015 Accepted: February 28, 2016 Published online: May 17, 2016

Correspondence: Jing Tian, MD. Department of Rheumatology, Second Xiangya Hospital, 410011 Changsha, China.

Tel: 86-073185295255 e-mail: yintianm@126.com

(02016 Turkish League Against Rheumatism. All rights reserved. 

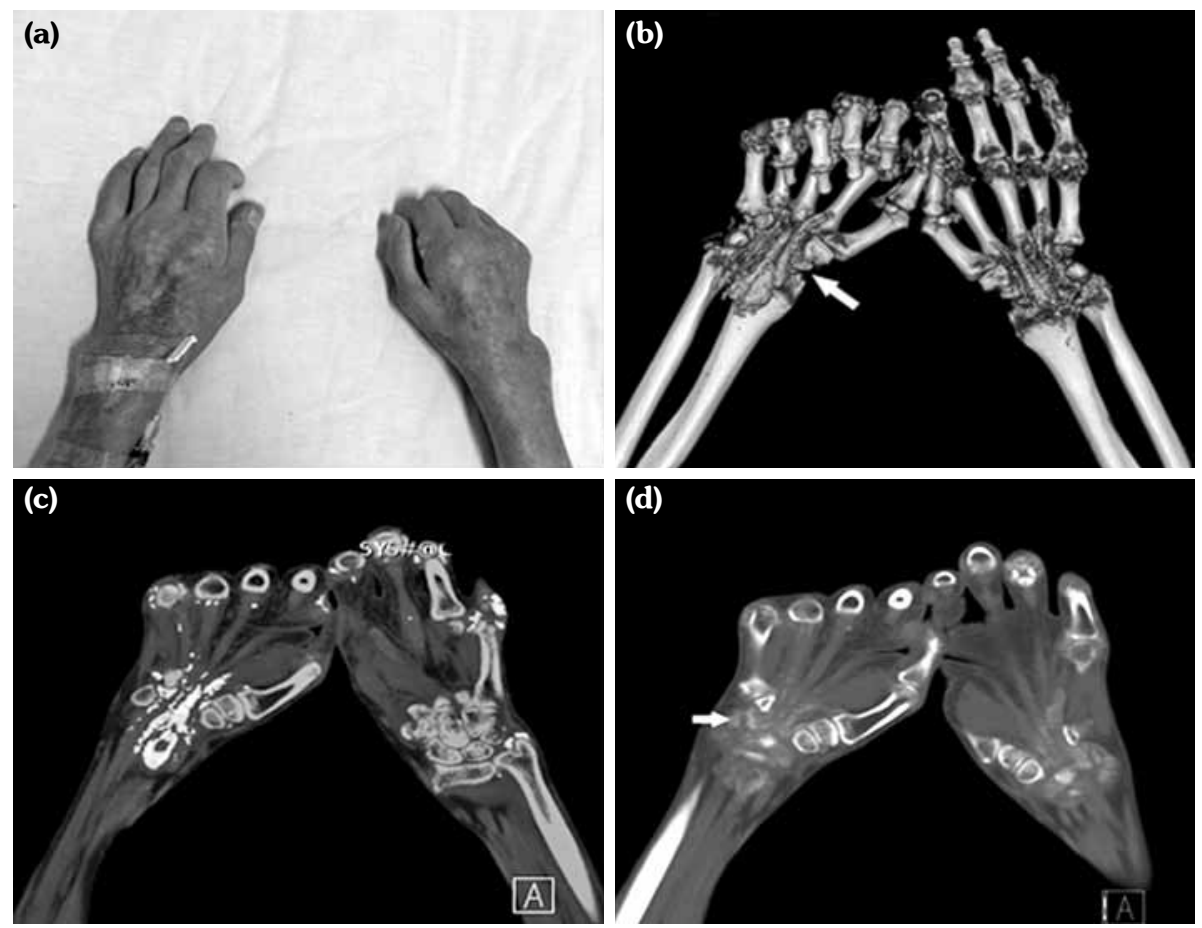

Figure 1. Carpal tunnel syndrome caused by gouty tophi. (a) Flexion deformity of both metacarpophalangeal and interphalangeal joints. (b, c) On three-dimensional reconstruction, extensive monosodium urate crystal deposits were located in intercarpal and radiocarpal joints with compression overlying flexor tendons by dual energy computed tomography (indicated by arrow). (d) Punched-out bony erosions were observed in carpal bones (indicated by arrow).

nerve, especially in the right hand, which supported the diagnosis of CTS. Furthermore, DECT showed extensive monosodium urate crystals in the intercarpal and radiocarpal joints with compression overlying the flexor tendons (Figure 1b, c). We also observed punched-out bony erosions in the carpal bones (Figure 1d). These aspects indicated severe and typical CTS caused by gouty tophi, which was further confirmed by biopsy of fine-needle aspiration (Figure 2). Surgery was recommended, but the patient refused to undergo surgery and chose to receive febuxostat. Uric acid level had decreased in the next six-month follow-up with little improvement for his numbness and deformity. A written informed consent was obtained from the patient.

\section{DISCUSSION}

Carpal tunnel syndrome is a common peripheral nerve entrapment syndrome. Carpal tunnel syndrome caused by gouty tophi is not rare, its incident reported to be approximately $0.6 \% .^{3}$ However, in the past, it was difficult to establish

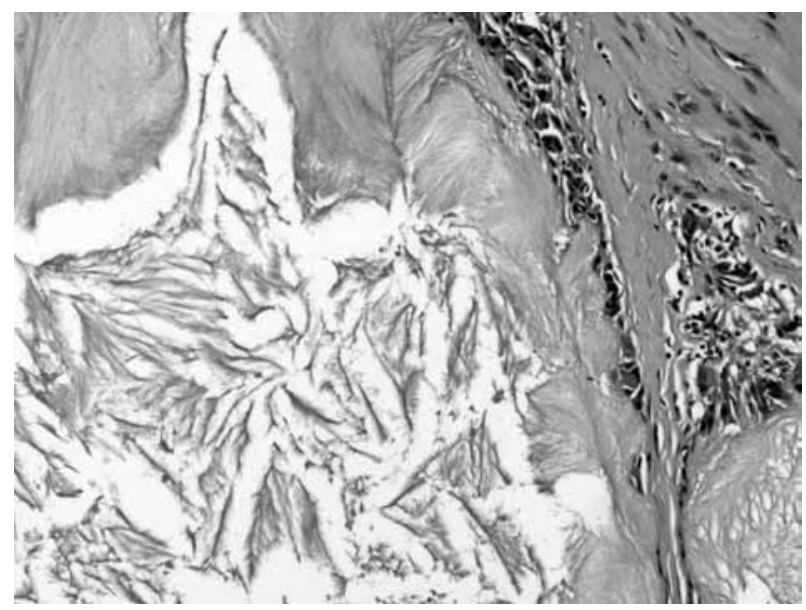

Figure 2. Hematoxylin-eosin staining of tissues from fine needle aspiration. Amorphous or needlelike acellular deposits were surrounded by chronic inflammatory reaction with abundant multinucleated giant cells (H-E x 100). 
a direct diagnosis unless biopsy or surgery was performed. In this condition, it is urgently required to conduct an easily applicable and non-invasive method. Dual energy computed tomography seems to be a very useful alternative since it can easily and directly show urate crystal deposition regardless of position. Thus it is more effective compared to ultrasound. Furthermore, it is a noninvasive method compared to biopsy. ${ }^{6}$

In our case, we successfully employed DECT to confirm the diagnosis and observed extensive monosodium urate crystal deposit in the intercarpal and radiocarpal joints with compression overlying the flexor tendons in a non-invasive way. Our experience indicates that DECT may be a good choice for the diagnosis of CTS associated with gouty tophi.

We recommended surgery to decompress the median nerve and improve the rigidity of flexor tendon. However, the patient decided to receive febuxostat because he could not afford surgical treatment. Follow-up study showed decreased urate level but his numbness and deformity did not improve. It is unfortunate that only a minority of gout patients receive effective treatment, although gout is believed to be cured by effective uratelowering treatment, particularly in developing countries. Early diagnosis and management of gout are important to avoid severe and irreversible complications. Gouty tophi should be considered as a possible cause of carpal tunnel syndrome if there is evidence of extrinsic compression of median nerve or symptoms originating from carpal tunnel.

\section{Declaration of conflicting interests}

The authors declared no conflicts of interest with respect to the authorship and/or publication of this article.

\section{Funding}

This study is supported by Hunan Provincial Natural Science Foundation of China (No. 14JJ3035 and 14JJ3036).

\section{REFERENCES}

1. Smith E, Hoy D, Cross M, Merriman TR, Vos T, Buchbinder $\mathrm{R}$, et al. The global burden of gout: estimates from the Global Burden of Disease 2010 study. Ann Rheum Dis 2014;73:1470-6.

2. Doherty M, Jansen TL, Nuki G, Pascual E, Perez-Ruiz F, Punzi L, et al. Gout: why is this curable disease so seldom cured? Ann Rheum Dis 2012;71:1765-70.

3. Rich JT, Bush DC, Lincoski CJ, Harrington TM. Carpal tunnel syndrome due to tophaceous gout. Orthopedics 2004;27:862-3.

4. Kim HS. Carpal tunnel syndrome caused by tophaceous gout. Korean J Intern Med 2014;29:544-5.

5. Hernández-Cortés P, Caba M, Gómez-Sánchez R, Gómez-Morales M. Digital flexion contracture and severe carpal tunnel syndrome due to tophaceus infiltration of wrist flexor tendon: first manifestation of gout. Orthopedics 2011;34:797-9.

6. Desai MA, Peterson JJ, Garner HW, Kransdorf MJ. Clinical utility of dual-energy CT for evaluation of tophaceous gout. Radiographics 2011;31:1365-75. 